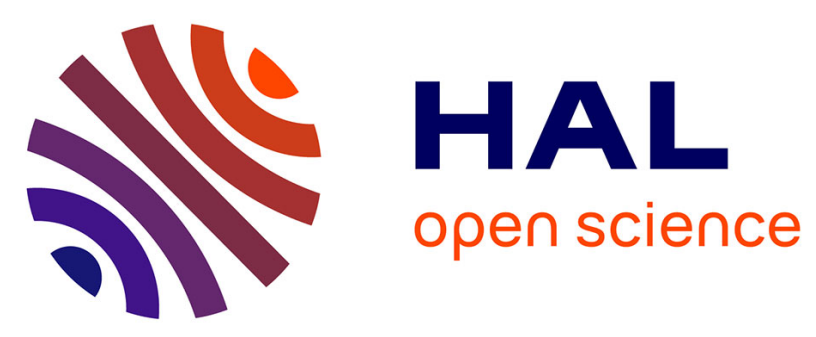

\title{
3D techniques to create interactive virtual museums: The state of the art in the epoch noe
}

\author{
Denis Pitzalis, Christian Lahanier, Ruven Pillay, Karina \\ Rodriguez-Echavarria, David B. Arnold
}

\section{- To cite this version:}

Denis Pitzalis, Christian Lahanier, Ruven Pillay, Karina Rodriguez-Echavarria, David B. Arnold. 3D techniques to create interactive virtual museums: The state of the art in the epoch noe. 2nd International Conference on Computer Graphics Theory and Applications, Mar 2007, Barcelone, Spain. pp.197-203, 10.5220/0002085201970203 . hal-00839848

\section{HAL Id: hal-00839848 \\ https://hal.science/hal-00839848}

Submitted on 1 Jul 2013

HAL is a multi-disciplinary open access archive for the deposit and dissemination of scientific research documents, whether they are published or not. The documents may come from teaching and research institutions in France or abroad, or from public or private research centers.
L'archive ouverte pluridisciplinaire HAL, est destinée au dépôt et à la diffusion de documents scientifiques de niveau recherche, publiés ou non, émanant des établissements d'enseignement et de recherche français ou étrangers, des laboratoires publics ou privés. 


\title{
3D TECHNIQUES TO CREATE INTERACTIVE VIRTUAL MUSEUMS: THE STATE OF THE ART IN THE EPOCH NOE
}

\author{
Denis Pitzalis, Christian Lahanier and Ruven Pillay \\ Centre de Recherche et de Restauration des Musées de France, \\ 14 quai François Mitterrand, Palais du Louvre, Porte des Lions, 75001 Paris, France \\ \{denis.pitzalis,christian.lahanier,ruven.pillay\}@culture.fr
}

Karina Rodriguez-Echavarria and David B. Arnold

University of Brighton

234 Watts Building, Moulseccomb, Brighton, United Kingdom

$\{k . r o d r i g u e z, d . a r n o l d\} @$ brighton.ac.uk

Keywords: Interactive Environments, Real-time Graphics, Graphics Application Pipeline, Technology in Museums

\begin{abstract}
Information and multimedia, such as images or 3D models, stored in databases are very important to preserve the information about historical artefacts and works of art. Nevertheless, the potential of digital content in databases is not fully exploited until it is used to create interactive ways to communicate to non $\mathrm{CH}$ specialist the significance that these objects have. 3D virtual environments are a suitable mechanism for giving context to, otherwise isolated, pieces of information. To achieve this, different techniques for 3D acquisition, integration and visualisation must work together in order to create 3D interactive virtual environments which are engaging and accessible for the visitors of a museum. In this paper we will describe the state of the art of the techniques for achieving this type of environments within the partners of the EPOCH Network of Excellence.
\end{abstract}

\section{INTRODUCTION: WHY BUILDING VIRTUAL MUSEUMS?}

The role of museums is shifting from that of an institution which mainly collects and stores artefacts and works of art towards a more accessible place where visitors can experience heritage in more engaging and interactive ways. As such, information and communication technologies have great potential for assisting not only in the documentation and preservation of information, such as images or 3D models about historical artefacts and works of art; but also for creating interactive ways to communicate to non $\mathrm{CH}$ specialists the significance that these objects have.

The concept of technology as another medium for representing heritage extends to all digital technologies in museums. For example: audiovisual guides, interactive multimedia screens and 3D interactive virtual environments. These mediums potentially provide a better understanding by contextualising artefacts in the museum in time and space and complement other forms of more traditional media, such as books, artist reconstructions, or photography, dealing with representations of the past (Thornton and
Rodriguez-Echavarria, 2006). In particular, 3D interactive virtual environments are a unique medium for representing heritage as they are able to provide a semi-realistic representation of 3 dimensional objects/spaces. This makes them a suitable mechanism for giving context to, otherwise isolated, pieces of information or physical objects which, at the moment, are displayed in glass cabinets or on museum walls. Hence, they enable visitors to interact virtually with historical objects, which will not be otherwise accessible due to their nature and fragility (Lahanier et al., 2002).

It has to be recognised, however, that the creation of Virtual Museums based on existing digital databases is more viable for larger Cultural Heritage institutions, who have more resources for the digitisation process, digital databases and the types of installation that virtual environments require. Medium and smaller institutes might find that the use of other mechanisms for representation are more suitable, such as audio-video story telling or much simpler multimedia applications. Nevertheless, it is expected that as the price of digitisation drops, and the cost of acquisition and maintenance of hardware and software becomes more accessible, more museums will increasingly be able to experiment with this type 
of environment.

The process of building a 3D virtual museum draws on many different technologies and digital sources. From the perspective of the Computer Graphics professional, technologies such as photogrammetry, scanning, modelling, visualisation, user interfaces, run-time engines and interaction techniques need to all work together. Additionally, the data exchange formats become essential to ensure that the digital sources are seamlessly integrated in the final experience. These domains need to interoperate efficiently and transparently in order to create engaging ways of presenting information.

This paper will present the state of the art of such techniques for creating 3D virtual museums within the partners of the EPOCH Network of Excellence (EPOCH, 2002). In addition, it will report on some concerns with, and propose some solutions for, the integration of the technologies previously mentioned. A case study proposed by the Centre de Recherche et de Restauration des Muses de France (C2RMF) will be presented to illustrate the integration of real tools for digitising and creating a 3D virtual environment, which takes advantage of the 3D documentation of works of art.

\section{DIGITISATION PROCESS}

Museum artefacts can vary greatly. They can be, for example, books, documents, statues, paintings and works of arts. As such, they are all made of different materials, with different shapes and sizes. It is also necessary to consider other types of 3D Cultural Heritage content, such as terrain data, architectural sites, virtual avatars, flora, fauna, as well as other types of content (i.e. video and sound), in order to portray more effectively the historical context of an object. Hence, different techniques for digitisation are required. The following subsection will describe three techniques, different in terms of cost, time and methodology, which are, at the moment, the most widely used means of capturing digitally the objects of a museum: photogrammetry, 3D laser scanning and modelling.

\subsection{Photogrammetry}

The goal of this system is to reconstruct a $3 \mathrm{D}$ object from a sequence of geometrical and colour calibrated images. The method used at the C2RMF is called shape from silhouettes (Hernandez-Esteban and Schmitt, 2003). The 3D digital photography capture system, developed in 2000 for the ACOHIR project (Martinez et al., 2000) consists of an accurate turntable to support the object, a simple digital camera, a professional lighting system and a PC to synchronise the acquisition. The computer controls the step by step rotation of the turntable, the lighting, the digital capture and the transfer of the files to the hard disk. At each step a digital image of the object is saved in sRGB in high definition (4000x5000 pixels) (Figure 1). The number of angular steps per rotation (usually 24 or 36 steps) can be changed. For complex objects several sequences can be acquired at various inclination angles of the camera to get a more complete panoramic view. A colour chart image is then used to calibrate the camera and to correct the colour of images.

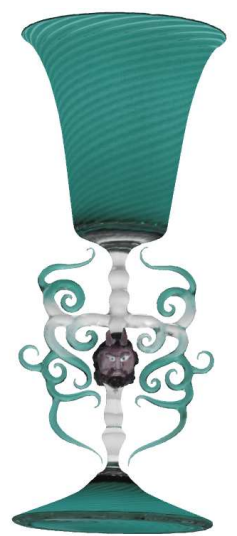

Figure 1: Glass vase, Buballo Lucio (Venice), Musée de Sèvres, inv. MNC27731. 3D model obtained by photogrammetry process.

When the set-up is complete for a given set of objects with similar shape and size (camera positioning for distance and inclination, zooming and focus, lighting) we proceed to a geometric calibration of the acquisition system before the object capture. The calibration can be performed in two ways: the first one, more secure but slower, is by acquiring several digital images of a black geometric target containing bright circular patches and by placing it in different positions and orientations on the turntable; the second one is entirely based on the silhouettes extracted from the image.

The use of silhouettes requires an accurate extraction of the object from the background, which is not always an easy task. The calibration allows us to obtain high resolution image sequences with a strictly controlled geometry. From the calibrated sequence of an object we can reconstruct a 3D model of the object with its colour texture. To do so, we use several types of information contained in the images. We first extract from each original colour image the silhouette of 
the object. Then we build in the 3D space a volume called the visual hull which is an upper-boundary of the real object volume. The visual hull is then carved in order to recover the missing concavities of the object surface. At the end of the process we are able to recover the apparent contours of the model. Finally, a texture map is computed from the original images for the reconstructed 3D model (Lahanier et al., 2004).

Another tool available for photogrammetric reconstruction is the EPOCH 3Dmodel web service (Vergauwen and Gool, 2006). This tool allows users to upload digital images to a service where a partial 3D model of the scene is reconstructed using shadowbased photogrammetry techniques.

\subsection{D laser scanning}

A more expensive way to digitise an object is by active 3D laser scanning. The fast capture time of a view (from 150,000 to 300,000 triangles with a Minolta VI900) allow the user to take many digital shots to cover all parts of the object. If the object is small, we can use a normal turntable to change it's orientation more quickly. The resolution of the MINOLTA camera is $100 \mu$ for $\mathrm{x}$ and $\mathrm{y}$ and $50 \mu$ in depth (Lahanier et al., 2005). The texture is acquired simultaneously by means of an internal video camera without colour calibration. The reconstruction of the object can be performed using MeshLab (MeshLab, 2006). Mesh$\mathrm{Lab}$ is an open source system for editing, cleaning, healing, inspecting, rendering and converting unstructured 3D triangular meshes.

Recently a new system has been tested at the C2RMF to scan paintings (Figure 2). This technique, developed by the Canadian National Research Council is based on the direct acquisition by multiple lowpower lasers (Taylor et al., 2002). The system scans a small (less than $100 \mu$ diameter) white laser spot from three (RGB) laser sources over the complete surface of an artefact, for example a painting. A triangulation based detection system simultaneously records the spatial measurements $(x, y, z)$ and the colour reflectance (RGB) from the spot. The maximum lateral resolution is of $50 \mu$ with a depth resolution of $10 \mu$.

A unique feature of this technology is the ability to examine the surfaces and roughness of paintings as well as the overall shape of canvas and panel paintings. The shape data recorded by the scanner originates from the immediate surface of the paint layer, under the varnish, rather than from the varnish surface. This results in a detailed high-resolution recording of the surface relief or 3D structure of the paint layer from brush stroke details as well as from craquelure formations due to ageing.

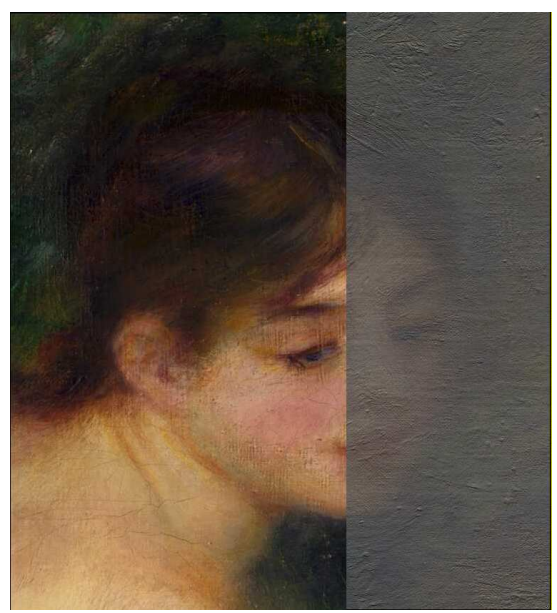

Figure 2: Naked woman, detail, Renoir Pierre Auguste, Museum of l'Orangerie, inv. RF 1963-13. 3D model obtained by color laser scan.

\subsection{Modelling}

Modelling is another technique where 3D polygonal representations of object are constructed either by hand or algorithmically, which includes generative and procedural modeling. Techniques for algorithmic modelling include: generative (Berndt et al., 2005a; Berndt et al., 2005b), procedural and rule based (Wonka et al., 2003; Mueller et al., 2006; Mueller et al., 2005). Other techniques are (Platings and Day, 2004) for landscape and (Ciechomsky et al., 2005; Ryder et al., 2005; Thalmann et al., 2005; Wilkinson et al., 2004) for avatars modelling (Figure 3).

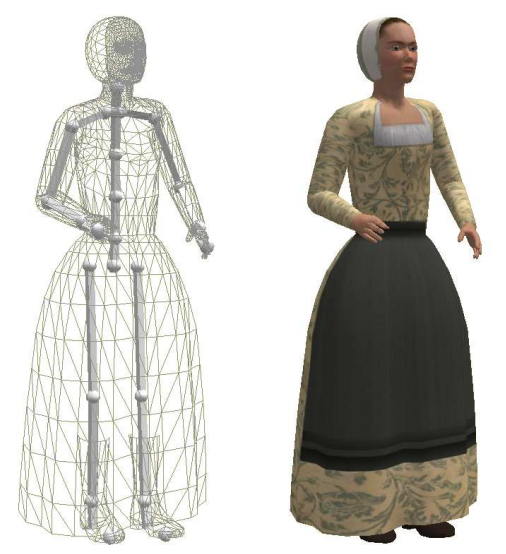

Figure 3: Animating Virtual Humans Modelling example. 


\section{INTEGRATION OF THE 3D DIGITISED CONTENT}

Once the artefacts have been digitalised, it is necessary to integrate them with other $\mathrm{CH}$ content and add interactivity. To do this different techniques are available and this is the stage where the integration of the different technologies is most critical.

The decision of which objects, content and interactivity to include is tightly coupled with the target public and the requirements of the application. One of the critical aspects of this stage is to ensure content reusability. This is especially the case as the role of technology in museums shifts towards achieving a "flexhibit" configuration approach (Tolva, 2006). In this approach, content is used in reconfigurable spaces, such as immersive virtual environments, multimedia, on the web, or on mobiles, by using different types of presentation technologies. Hence, reusability is essential as it will provide maximum flexibility and will avoid repeating the work for use within different contexts. To automate this, previous approaches include the use of presentation domains (Patel et al., 2003), which correspond to different environments in which the digital artefacts can be used.

Cultural Heritage 3D interactive environments have normally been handcrafted (Calori et al., 2003; Gaitatzes et al., 2004; Ch'ng et al., 2004) using device-independent graphics libraries (e.g. OpenGL (OpenGL, 2006)), scene graph based libraries (e.g. OpenSG (OpenSG, 2006), OpenScenegraph (OpenSceneGraph, 2006), Performer (Performer, 2006)), game engines for integrating content (e.g. Unreal Tournament (UT, 2006), Aurora toolkit (NWN, 2006)) or 3D authoring tools (Rodriguez-Echavarria et al., 2005; Laycock and Day, 2003). Although this affords a great degree of freedom, it also requires experienced graphics professionals as developers (Figure 4). As a consequence museum curators or other $\mathrm{CH}$ professionals who may not have this experience, are unable to participate fully and the development focus can be diverted from creativity to technology. For this reason, it is important to involve curators and museums designers at this critical stage of the development process.

Integration also requires the translation of content data formats from the original applications to a format that can be used to stitch content together. Almost all applications for creating content rely on proprietary data formats that can only be reused by making translations which require inefficient modifications to the files. Although a lot of work has been done in this area, since the first attempts to define ISO standards for computer graphics (Arnold and Bono,

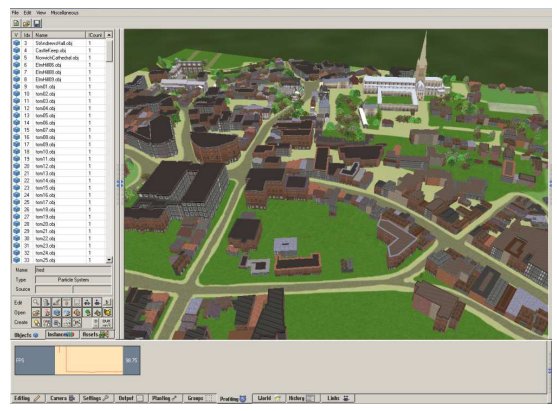

Figure 4: Authoring tool example.

1988), there is still a lack of international commonly accepted standards for exchanging 3D data, such as generalised scene topology, individual models and behavioural mechanisms, as well as offering flexibility for custom components. This is specially important for Cultural Heritage artefacts in museums, where the conservation of the data well beyond the lifetime of current systems is essential. Currently, X3D (succeeding the earlier VRML) and COLLADA (COLLAborative Design Activity) are two popular standards for encoding 3D objects into common formats for sharing between applications. COLLADA is increasingly used in 3D content creation whilst X3D has particular legacy value and familiarity in the field. Both formats can encode a scene using an XML syntax. Some of their main differences are:

- X3D (Web3D Consortium 2006) is an ISO standard, while COLLADA was originally established by Sony and then adopted by other main players in the gaming industry. These will have an effect on which applications will take advantage of these formats as they are being directly supported by the tool vendors.

- COLLADA is designed as an interchange format, while X3D is designed as a content deployment format, targeting web type applications.

\section{VISUALISATION OF 3D INTERACTIVE VIRTUAL MUSEUMS}

Technologies for visualisation are constantly being developed and at the moment no single technology can fulfil all applications. Such technologies vary in size, shape, stereo capability, cost, type of view (direct view vs. projection) and their usage depends on the specific characteristics of the installation. Recently, popular visualisation installations in museums 
have focused on single or multiple projection architectures, including CAVE based environment (Rapp and Weber, 2005; Christopoulos et al., 2001). These environments usually consist of projection screen installations, stereo surround sound and other devices for users to interact with the environment. Another type of installation consisting of a video wall using direct view screen technologies instead of projection systems is starting to emerge and is also gaining popularity. The visualisation of 3D interactive virtual museums provides a set of unique challenges for visualisation technology, as this types of environments:

- Integrates content from a mixture of acquisition techniques and thus may contain a mixture of large meshes.

- Require interactive frame rates for the visitors to engage with the application.

- Require different rendering techniques in case different modelling approaches had been used.

Techniques such as cluster based visualisation might be a solution for this need. This technique involves a cluster of standard PC's driving an array of projectors or screens, where each PC is responsible for rendering a predefined part of the display. The reason for its suitability are mainly:

- the low hardware and software acquisition and maintenance costs

- the power of modern graphics cards, driven by the computer video games industry, now rival the more powerful graphics engines.

By using this technique, a layer of software is still necessary above the operating system and below the application level to provide synchronisation of input devices and the $3 \mathrm{D}$ environment. For this, the mechanisms that are available include: OpenSG, Chromium (Chromium, 2006) and Equalizer (Equalizer, 2006).

\section{A CASE STUDY: GALLO-ROMAN WHITE CLAY FIGURINES}

During the Gallo-Roman period (from 40 to $300 \mathrm{AD}$ ), numerous workshops in the Centre of Gaul produced white clay figurines made of kaolin. More than 5,800 figurines are registered in the EROS database at the C2RMF(Aitken et al., 2005). The knowledge acquired on the production of these artefacts and the extension of its diffusion confirms the role of the ancient commercial routes of the Roman Empire and the religious influence between civilisations from the Northern and Southern French countries.

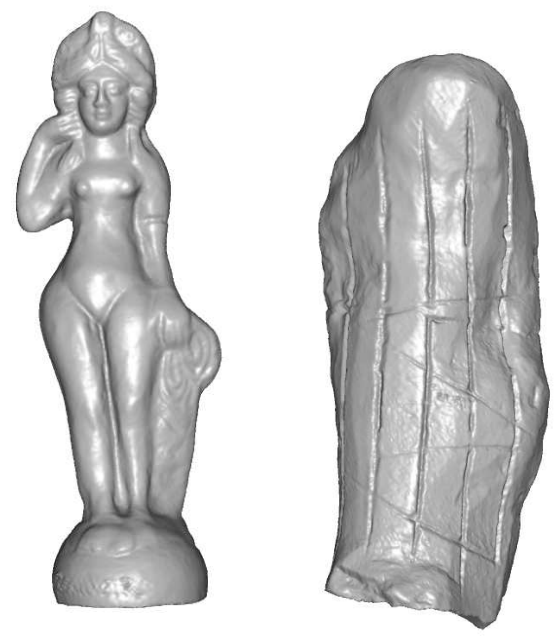

Figure 5: A "Venus Anadyomene" and it signed mould. Anne de Beaujeu Museum, Moulins, France

The figurines production starts by the modelling of a "genotype" or "archetype" made by the "coroplathe". Then, by casting the genotype, its shape was extracted using prints to make moulds; the number of which depended of the complexity of its shape ( 3 for a Venus comprising the two pieces for the front, the back and the base; 13 pieces for a Spinario). The several moulds extracted from a genotype are generally signed on their outer part to identify the name of the producer or that of the moulder. Then, after drying the moulds, a great number of copies can be stamped. The different pieces are joined and glued with diluted clay called "barbotine" to rebuilt the empty figurines similar to the genotype. In order to prevent the model from exploding during the firing process, a small hole is made in the back of the fresh ceramic to evacuate the pressure of the vapour. During the drying and the baking process the objects shrink. When the genotype and the moulds are worn, new castings are made using the existing figurines. This process produces generations of artefacts which are progressively smaller (approximately 10\% reduction each time). To study the production and the spread of these artefacts throughout Gaul and in the Roman Empire, archaeologists have built a typology of the different kinds of God, Goddess, figures, animals, fruits, aliments and toys, etc. As the objects were dispersed and broken, the classification consisted of designing and describing the incomplete models. It was a single historian that had to perform this classification in order for it to be coherent due of the subjective visual aspect of this method without 3D measurement. To automate this process, an electronic inventory and 3D modelling were coupled with the chemical analysis of the 
clay made at the C2RMF on few thousands of the figurines.

One of the focal points of the French national project "Metamorphose" was to present C2RMF results to the public in an exhibition. The use of a Virtual Museum was a suitable solution to this need, as most of the figurines were already digitised and classified in a database. The digitisation involved laser scanning techniques for the artefacts and modelling techniques for the virtual environment (Figure 6). The application was rendered using OpenGL and visualised by the users using a single PC display. The main objective of the application was to contextualise the reconstructed 3D figurines in a virtual environment representing the original workshop. The reason for this, was to show the visitors not only the figurines but also, their history and characteristics as well as the processes used for their production. This allowed visitors to experience how the past might have been for that particular society, and understand the production techniques as well as gain new knowledge of the era.

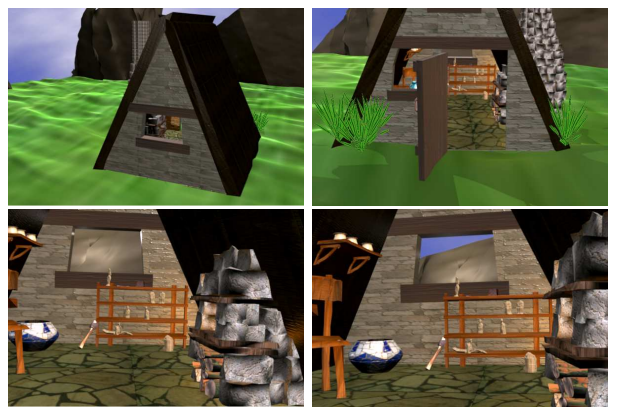

Figure 6: Animating Virtual Humans Modelling and Authoring tool example.

\section{CONCLUSIONS}

Within the Cultural Heritage domain access is so limited that the opportunity to create digital representations is precious. For this reason, it is crucial that content already existent should be fully integrated into effective means of presentation. This paper highlighted the importance of using information and communication technology as a more effective media for representing heritage. Although, it was recognised that museums which already have databases with digital artefacts might take better advantages of creating this type of environments for visitors to better appreciate the artefacts' value. The paper presented an overview of the techniques which are available for creating a 3D interactive virtual museum. The different techniques for digitisation, integration and visuali- sation were described. In addition, several issues with the current development cycles of interactive experiences were identified. These issues might not only apply to the Cultural Heritage application area, but for many others; hence, its consideration is important. The paper highlighted the importance of the content integration stage of the process and the need for common guidelines as well as the adaptation of general purpose standards, which are necessary for all different techniques to work together efficiently. Finally, the potential of using content in existing databases, such as the C2RMF EROS database, was used as an example of how virtual environments is an area which is still to have a greater impact on the way that visitors experience museums today.

\section{ACKNOWLEDGEMENTS}

Part of this work has been conducted as part of the EPOCH Network of Excellence (IST-2002-507382) within the IST (Information Society Technologies) section of the Sixth Framework Programme of the European Commission.

\section{REFERENCES}

Aitken, G., Lahanier, C., Pillay, R., and Pitzalis, D. (2005). Eros: An open source database for museum conservation restoration. In 14Th Triennial Meeting ICOM$C C$, The Hague, Netherlands.

Arnold, D. and Bono, P. (1988). Cgm and cgi: Metafile and interface standards for computer graphics. In Springer-Verlag, Heidelberg New York.

Berndt, R., Fellner, D., and Havemann, S. (2005a). Generative $3 \mathrm{~d}$ models: a key to more information within less bandwidth at higher quality. In Web3D.

Berndt, R., Gerth, B., Havemann, S., and Fellner, D. (2005b). 3d modeling for non-expert users with the castle construction kit. In 6th International Symposium on Virtual Reality, Archaeology and Intelligent Cultural Heritage, Pisa, Italy.

Calori, L., Diamanti, T., Guidazzoli, A., Liguori, M., Mauri, M., and Valentini, L. (2003). Certosa virtual museum: a dynamic multilevel desktop vr application. In Eurographics, Granada, Spain.

Ch'ng, E., Stone, R. J., and Arvanitis, T. N. (2004). The shotton river and mesolithic dwellings: Recreating the past from geo-seismic data sources. In 5th International Symposium on Virtual Reality, Archaeology and Intelligent Cultural Heritage, Brussels, Belgium.

Christopoulos, D., Gaitatzes, A. G., and Roussou, M. (2001). Reviving the past: Cultural heritage meets virtual reality. In 2nd International Symposium on 
Virtual Reality, Archaeology, and Cultural Heritage, Athens, Greece.

Chromium (2006). http://chromium.sourceforge.net/.

Ciechomsky, P., Schertenleib, S., Maim, J., Maupu, D., and Thalmann, D. (2005). Real-time shader rendering for crowds in virtual heritage. In 6th International Symposium on Virtual Reality, Archaeology and Intelligent Cultural Heritage, Pisa, Italy.

EPOCH (2002). Network of excellence, ist-2002-507382: http://www.epoch-net.org.

Equalizer (2006). http://www.equalizergraphics.com/.

Gaitatzes, A., Christopoulos, D., and Papaioannou, G. (2004). The ancient olympic games: Being part of the experience. In 5th International Symposium on Virtual Reality, Archaeology and Intelligent Cultural Heritage, Brussels, Belgium.

Hernandez-Esteban, C. and Schmitt, F. (2003). Silhouette and stereo fusion for a $3 \mathrm{~d}$ object modelling. In 4 th International Conference on 3D Digital Imaging and Modelling, Banff, Canada.

Lahanier, C., Aitken, G., Cotte, P., Pillay, R., and Pitzalis, D. (2004). Digital capture of archive, objects and paintings for research and conservation. In Electronics and Visual Arts, Florence, Italy.

Lahanier, C., Aitken, G., J.Shindo, Pillay, R., Martinez, K., and Lewis, P. (2002). Eros: An open source multilingual research system for image content retrieval dedicated to conservation-restoration exchange between cultural institutions. In 13th Triennial Meeting, ICOM-CC, Rio de Janeiro, Brasil.

Lahanier, C., Feihl, O., Jeanlin, M., Pitzalis, D., and Schmitt, F. (2005). 3d modelling of archaeological objects for conservation, visualisation, colour and shape characterisation, details comparison. In 14Th Triennial Meeting ICOM-CC, The Hague, Netherlands.

Laycock, R. and Day, A. (2003). Rapid generation of urban models. Computers \& Graphics.

Martinez, K., Cupitt, J., and Perry, S. (2000). Object browsing using the internet imaging protocol. In Computer Networks and ISDN Systems.

MeshLab (2006). http://meshlab.sourceforge.net/.

Mueller, P., Vereenooghe, T., Ulmer, A., and Gool, L. V. (2005). Automatic reconstruction of roman housing architecture. In Recording, Modeling and Visualization of Cultural Heritage.

Mueller, P., Wonka, P., Haegler, S., Ulmer, A., and Gool, L. V. (2006). Procedural modeling of buildings. In ACM SIGGRAPH 2006 / ACM Transactions on Graphics.

NWN (2006). Aurora toolkit: http://nwn.bioware.com/builders/.

OpenGL (2006). http://www.opengl.org/.

OpenSceneGraph (2006). http://www.openscenegraph.org/. OpenSG (2006). http://www.opensg.org/.

Patel, M., White, M., Walczak, K., and Sayd, P. (2003). Digitisation to presentation -building virtual museum exhibitions. In Vision, Video and Graphics, Bath, UK.
Performer (2006). http://www.sgi.com/products/software/performer/.

Platings, M. and Day, A. (2004). Compression of large scale terrain data for real time visualization using a tiled quad tree. In Computers Graphics Forum.

Rapp, S. and Weber, I. (2005). Lumenactive: A novel presentation tool for interactive installations. In 6th International Symposium on Virtual Reality, Archaeology, and Cultural Heritage, Pisa, Italy.

Rodriguez-Echavarria, K., Genereux, M., Arnold, D., Day, A., and Glauert, J. (2005). Multilingual virtual city guides. In Computer Graphics and Applications, Novosibirsk, Russia.

Ryder, G., Flack, P., and Day, A. (2005). A framework for real-time virtual crowds in cultural heritage environments. In 6th International Symposium on Virtual Reality, Archaeology and Intelligent Cultural Heritage, Pisa, Italy.

Taylor, J., Beraldin, J. A., Godin, G., Cournoyer, L., Rioux, M., and Domey, J. (2002). Nrc 3d imaging technology for museum and heritage. In 1st International Workshop on $3 D$ Virtual Heritage, Geneva, Switzerland.

Thalmann, N. M., Kim, H., Egges, A., and Garchery, S. (2005). Believability and interaction in virtual worlds. In IEEE Computer Society, Los Alamitos, CA, USA.

Thornton, M. and Rodriguez-Echavarria, K. (2006). Sociocultural impacts of virtual heritage in museums: an interdisciplinary analysis. In Heritage Impact International Symposium, Brighton, United Kingdom.

Tolva, J. (2006). Towards a participatory cultural environment: The evolution of virtual representation in the humanities. In 7th International Symposium on Virtual Reality, Archaeology and Intelligent Cultural Heritage, Nicosia, Cyprus.

UT (2006). Unreal tournement technology: http://www.unrealtechnology.com/.

Vergauwen, M. and Gool, L. V. (2006). Web-based 3d reconstruction service. In Machine Vision Applications.

Wilkinson, N., Jennings, V., Glauert, J., and Elliott, R. (2004). A speaking avatar toolkit for cultural heritage applications. In 5th International Symposium on Virtual Reality, Archaeology and Intelligent Cultural Heritage, Brussels, Belgium.

Wonka, P., Wimmer, M., Sillion, F., and Ribarsky, W. (2003). Instant architecture. In Siggraph. 